\title{
COVID-19 en población pediátrica y sus factores asociados
}

\section{COVID-19 and associated factors in the pediatric population}

Nohely L. Huapaya-Javier*, Mayra Teodoro-Gallardo y Luis Llanco-Albornoz

Escuela Profesional de Medicina Humana, Universidad Privada San Juan Bautista, Filial Chincha, Ica-Perú

Señor Editor,

Después de leer artículo de Rivas-Ruiz, et al. ${ }^{1}$, percibimos ciertos errores y sugerimos su atención. La literatura evidencia que existen diferencias en el proceso de la enfermedad por coronavirus 2019 (COVID-19) entre los lactantes, niños y adolescentes dentro del grupo pediátrico como lo reportaron Panahi, et al. ${ }^{2}$, donde los niños cursaban mayormente con fiebre y tos seca, mientras que los lactantes presentaron aumento de la frecuencia cardiaca. A su vez, hubiese sido más interesante analizar la variable edad en diferentes categorías a fin de conocer las diferencias de la presentación clínica y factores asociados a la mortalidad por esta enfermedad. En ese sentido, Bhopal, et al., al estratificar la tasa de mortalidad, observaron una marcada diferencia en niños de hasta el $0.28 \%$, mientras que en los adolescentes varió del 0.58 al $3.39 \%^{3}$. Por lo tanto, categorizar la edad nos permite identificar factores claves en la epidemiología de la infección por COVID-19 en la población pediátrica.

\section{Financiamiento}

Los autores declaran no haber recibido financiamiento para este trabajo.

\section{Conflicto de intereses}

Los autores declaran no tener conflicto de intereses.

\section{Bibliografía}

1. Rivas-Ruiz R, Roy-Garcia IA, Ureña-Wong K, Aguilar-Ituarte F, Vázquez-de Anda GF, Gutiérrez-Castrellón P, et al. Factores asociados a muerte en niños con COVID-19 en México. Gac Med Mex. 2020;156(6):526-32.

2. Panahi L, Amiri M, Pouy S. Clinical characteristics of COVID-19 infection in newborns and pediatrics: A systematic review. Arch Acad Emerg Med. 2020;8(1):1-8.

3. Bhopal SS, Bagaria J, Olabi B, Bhopal R. Children and young people remain at low risk of COVID-19 mortality. Lancet Child Adolesc Heal. 2021;5(5):12-3.
Correspondencia:

${ }^{*}$ Nohely L. Huapaya-Javier

E-mail: nohely.huapaya@upsjb.edu.pe

0016-3813/С 2021 Academia Nacional de Medicina de México, A.C. Publicado por Permanyer. Este es un artículo open access bajo la licencia CC BY-NC-ND (http://creativecommons.org/licenses/by-nc-nd/4.0/). 